Chimia 46(1992) 3-5

(C) Schweiz. Cheniker-Verband; ISSN 0009-4293

\section{Die Schweizerische Vereinigung diplomierter Chemiker HTL (SVCT)}

\author{
Urs Girard* (Präsident, Münsingen)
}

Abstract. Founded in Basel in 1946, the Swiss Association of Certificated Chemists HTL (SVCT) grew into a professional association which represents the specific interests of state engineering institute graduate chemists from throughout Switzerland. Thanks to its steady growth and a membership which embraces a substantial number of all chemists HTL in the country, the SVCT today is one of the most important chemists' associations in Switzerland.

Apart from its political activities for the profession, the SVCT is also involved in the promotion of the training and development of young chemists - it provides the engineering schools and students with expertise from its ranks to act as consultants and placement officers for job experience; it awards prizes; and it publishes an illustrated brochure, which is periodically updated, on the profession. A further major activity of the SVCT is the much respected series of conferences organized on a regular basis to consider not only the technical aspects of chemistry and related areas but also the human factor.

An information bulletin is also issued periodically to keep members informed about major events and activities. In addition, the SVCT conducts surveys among its members which serve as a state-of-the-art review and a touchstone for the future.

The SVCT cultivates a close collaboration with the authorities, industry, institutions of professional formation, and other professional associations. Particularly important in this context are the contacts with the Swiss Chemists' Association, the executive committee of which traditionally includes SVCT representatives, and whose current efforts to bring all chemists' associations in Switzerland closer together are supported by the SVCT.

\section{Die SVCT - wer ist das?}

Im Dezember 1946 gründeten Berufskollegen aus der Region Basel - Chemieabsolventen der damals Technikum genannten heutigen Ingenieurschulen (HTL) der Schweiz-die 'Vereinigung diplomierter Chemiker Schweizerischer Techniken (VCT)'.Ziel des Zusammenschlusses war es, der oftmals noch fehlenden oder nur teilweise vorhandenen Anerkennung als Chemiker bei den Behörden und Arbeitgebern vermehrt Geltung zu verschaffen. Da zu jener Zeit keine Institution bestand, welche die ganz spezifische Vertretung der Berufs- und Standesinteressen der Technikumschemiker wahmahm, erschien die Gründung eines eigenen Berufsverbandes notwendig.

Im Laufe der Zeit hat sich diese ursprünglich baslerische Vereinigung stark weiterentwickelt und laufend den neuen Gegebenheiten im tertiären Bildungswe-

*Korrespondenz: Urs Girard

Personaldienst Höhere Angestellte und wissenschaftliche Mitarbeiter, Ciba-Geigy AG $\mathrm{CH}-4002$ Basel sen und der Berufswelt angepasst. Gleichzeitig veränderte sich auch das Technikum immer mehr: vom Hochschulausbildungsersatz der Vorkriegszeit für den (ländlichen) Nachwuchs zur Fortbildungsinstitution für die Absolventen einer Berufslehre bis hin zur heutigen praxisorientierten eigenständigen Ingenieurschule. Dementsprechend wurden 1964 bei der Revision des Berufsbildungsgesetzes die Technika in 'Höhere Technische Lehranstalten (HTL)' umbenannt, was zur Folge hatte, dass 1967 auch der Verbandsname der VCT in 'Vereinigung diplomierter Chemiker HTL' geändert wurde.

Heute repräsentiert der Verband die Chemiker HTL der ganzen Schweiz (Fig. $I$ und 2), was sich seit 1974 im Namen 'Schweizerische Vereinigung diplomierter Chemiker HTL (SVCT)' ausdrückt. Der andauemde, starke Mitgliederzuwachs (Fig. 3), der hohe Mitgliedschaftsanteil aller Chemiker HTL und eine junge Alterstruktur (zwei Drittel sind nicht älter als vierzig) machen die SVCT heute zu einem Schweiz. der bedeutendsten Chemikerverbände der

\section{Welche Ziele verfolgt die SVCT?}

Die SVCT bezweckt die Wahrung und Förderung der Berufs- und Standesinteressen von diplomierten Chemieabsolventen der Ingenieurschulen (HTL). Insbesondere nimmt sie diese spezifischen Interessen dort wahr, wo dies andere Organisationen nicht oder nur teilweise können. Dabei vertritt sie, in Zusammenarbeit mit solchen Schwesterinstitutionen, auch die Interessen anderer Chemieabsolventen, speziell wenn es sich um gemeinsame oder das Ansehen der Chemie generell betreffende Anliegen handelt.

Die Zielsetzungen der SVCT umfassen somit u.a.:

- Vernehmlassungen zu und Einflussnahme auf Vorgänge, welche die Mitglieder in ihrer Berufsausübung berühren (Gesetze, Verordnungen etc.)

- Vermittlung von berufsrelevanten Infomationen

- Durchführung verbandsinterner und öffentlicher Veranstaltungen

- Beratung der Mitglieder in Berufsfragen und Förderung der gegenseitigen Beziehungen

- Kontakte mit den Ingenieurschulen, Unterstützung ihrer Anliegen

- Zusammenarbeit mit anderen Berufsverbänden, insbesondere im gemeinsamen Auftritt nach aussen, sowie mit Behörden und der Industrie.

\section{Was tut und bietet die SVCT?}

\subsection{Informationsvermittlung}

Über die wesentlichen Aktivitäten der SVCT und die Vorgänge im beruflichen Umfeld werden die Mitglieder durch ein periodisch erscheinendes Mitgliederorgan orientiert.

1983 wurde erstmals eine gesamtschweizerische Erhebung zur Population und Salärsituation der Chemiker HTL durchgeführt, die seither regelmässig wiederholt wird und deren Resultate den Mitgliedern, und auf Wunsch auch weiteren Interessenten, zur Verfügung gestellt werden. 1987 erfolgte zudem eine umfangreiche Mitgliederbefragung zur Zukunft des Chemikers HTL, in welcher Status und Zukunfttrends bezüglich Branchen, Firmen, Technologien, Tätigkeitsgebieten, Laufbahn, Aus- und Weiterbildung, Anforderungsprofil und beruflicher Zufriedenheit sowie zur Rolle von Berufsverbänden ermittelt wurden.

Periodisch gibt die SVCT ein aktuelles Mitgliederverzeichnis heraus, welches auch Studienort, Tätigkeitsgebiet und Firma oder Arbeitsinstitution der Mitglieder umfasst und so als Kontaktplattform genutzt werden kann. 


\subsection{Anlässe und Veranstaltungen}

Seit Anfang, d.h. seit 1968, beteiligt sich die SVCT mit einem aktuellen Themenkreis an der mit der Chemiefachmesse ILMAC verbundenen Tagungswoche des 'Basler Treffen für Chemische Technik'. Dabei bildet die SVCT-Vortragsreihe traditionsgemäss den Abschlusstag des Basler Treffens und behandelt nicht bloss die Technik, sondern diskutiert auch die Rolle des in der Chemie tätigen Menschen und die Interaktionen zu Gesellschaft und Mitwelt. Erfreulicherweise fand dieses Konzept bisher stets starke Beachtung und dementsprechend überdurchschnittliche Besucherbeteiligung, wobei der bisherige Höhepunkt eine mit Eidophor-Grossprojektion vor mehr als 1000 Teilnehmern übertragene Felddemonstration von Sicherheitsuntersuchungen war.

Auch die jährlichen Generalversammlungen werden in der Regel mit Begleitveranstaltungen oder Firmen- und Institutsbesuchen verbunden. Neben dem Einblick in die Verbandsgeschäfte erhalten die Mitglieder so häufig auch solche in weniger bekannte Tätigkeits- und Interessengebiete ihrer Berufskolleg(inn)en und können nicht zuletzt mit diesen gesell- schaftliche wie fachliche Kontakte aufbauen oder unterhalten.

Die ursprünglich verbandsinternen SVCT-Fachtagungen finden seit 1988 öffentlich und in Zusammenarbeit mit der chemischen Industrie statt. Von Anfang weg war auch diesen Anlässen ein grosser Erfolg beschieden, sodass sie seither zusätzlich zu den ILMAC-Tagungen regelmässig weitergeführt werden (Fig. 4).

\subsection{Berufs- und Standespolitik}

Die SVCT setzt sich ganz besonders dafür ein, dass der Chemiker HTL auf Gesetzesebene, in Verordnungen und Richtlinien von Behörden usw. seiner Ausbildung gemäss angemessen berücksichtigt wird. Dazu gehört die Beteiligung an entsprechenden Vernehmlassungen oder gegebenenfalls auch eine notwendige direkte Intervention bei den zuständigen Stellen. Zur Illustration mögen die nachfolgenden Beispiele dienen:

Seit ihrer Gründung bemühte sich die SVCT für eine ausbildungsgerechte $\mathrm{Be}$ rufsbezeichnung im Berufsbildungsgesetz, wobei erreicht werden konnte, dass die ursprünglich vorgesehene Bezeichnung 'Chemiker-Techniker HTL' in einer vor-

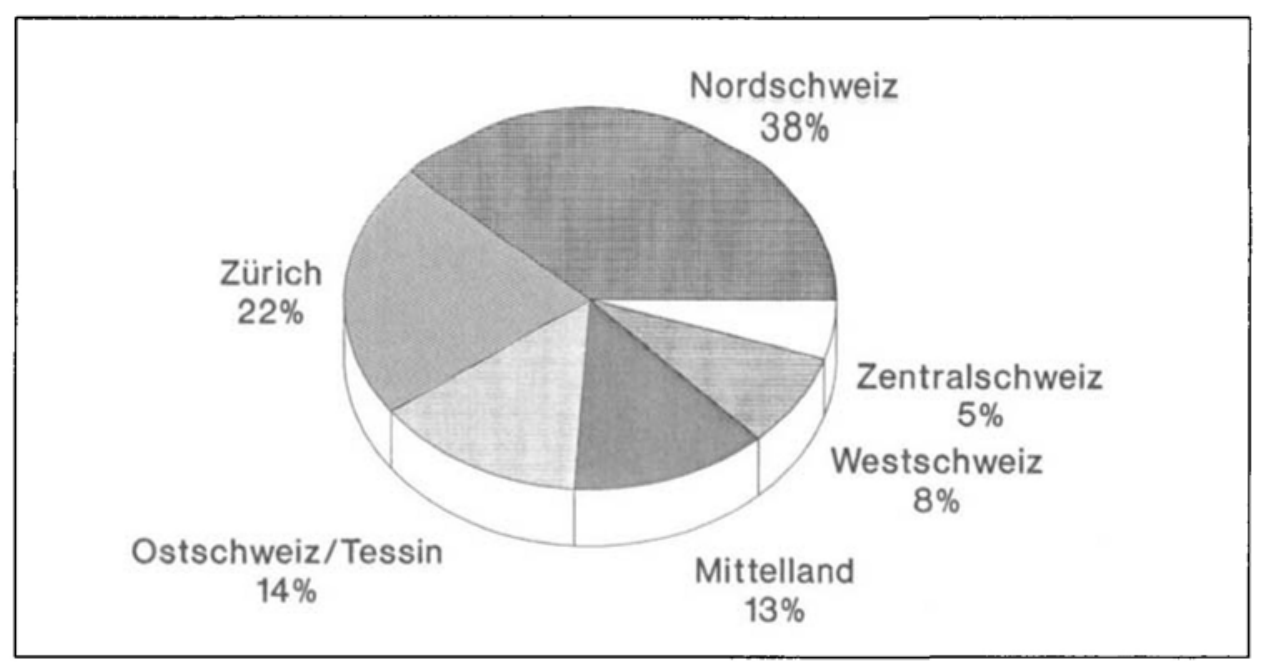

Fig. 1. Chemiker HTL Arbeitsregionen

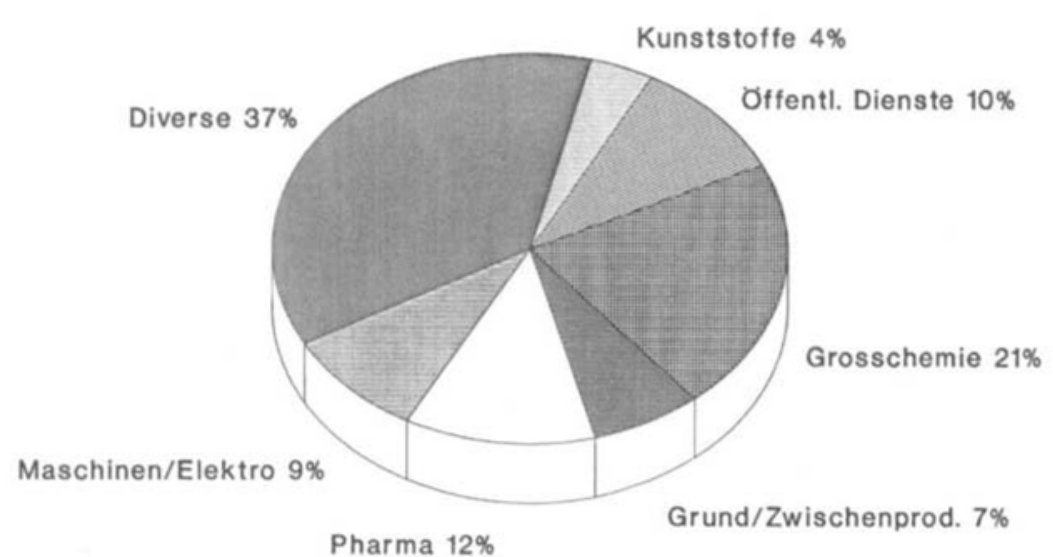

Pharma $12 \%$ gängiger Verordnung des EDI auf 'Chemiker HTL' abgeändert, bzw. gemäss bisheriger Usanz bestätigt wurde. Mit Unterstützung der SVCT erreichten später auch die übrigen Berufsverbände für ihre $\mathrm{Ab}$ solventen eine sinngemässe Gleichstellung in ihrer Berufsbezeichnung. Im Anschluss an einen nationalen Ingenieurtag von 1991, in welchem insbesondere auch auf die Stellung des HTL Ingenieurs im sich wandelnden Europa eingegangen wurde und an dessen Organisation sich die SVCT beteiligte, äusserte sich der schweizerische Bundesrat auf eine parlamentarische Anfrage hin schliesslich positiv zur bisher verwehrten Benutzung des Präfix 'diplomiert' in der Berufsbezeichnung der HTL-Absolventen.

Im Entwurf des Giftgesetzes von 1969 waren die Chemiker HTL nicht erwähnt, was ihnen im Umgang mit Giftstoffen in der täglichen Berufspraxis grosse Schwierigkeiten bereitet hätte. Bei der Revision der Vollzugsverordnung 1983 konnte die SVCT erreichen, dass der Chemiker HTL - über die inzwischen zugestandene Bewilligung $B$ hinaus - die nun uneingeschränkte Giftbewilligung A erhielt.

In verschiedenen Entwürfen der Richtlinien der IKS war der Chemiker HTL ebenfalls nicht berücksichtigt, wodurch ihm die Übernahme verantwortlicher Positionen in der pharmazeutischen und damit verwandten Industrie verwehrt geblieben wäre. Auch hier erreichte die SVCT die volle Gleichstellung der Chemiker HTL in den heute gültigen Verordnungen.

\subsection{Nachwuchsförderung und Kontak- pflege}

Ein besonderes Anliegen der SVCT ist, jungen, vor der Berufs- bzw. Studienwahl stehenden Menschen sowie weiteren Interessenten Einblick in das vielseitige Berufsleben des Chemikers HTL zu vermitteln und so Nachwuchs für die Chemie zu gewinnen.

$\mathrm{Zu}$ diesem Zweck wurde, zum ersten Mal 1960, das Berufsbild des Chemikers HTL in einer Broschüre mit dem Titel 'Der Chemiker HTL-Berufshild' zusammengestellt und in mehreren tausend Exemplaren für die Information des beruflichen Nachwuchses aufgelegt. 1969 und 1980 erfolgten dem neuesten Stand der Technik und der Ausbildung angepasste Neuauflagen dieser ansprechenden Publikation, welche 1992 in vierter Ausgabe erneut gesamtschweizerisch verteilt werden wird.

Die SVCT pflegt enge Kontakte zu den Ingenieurschulen und deren Chemie-Abteilungen. Sie steht ihnen sowie den Studenten als Partner und als Vermittler zur Berufspraxis, für die Stellungnahme zu aktuellen Fragen der HTL, bei Lehrplan-

Fig. 2. Chemiker HTL Arbeitgeber-Branchen 
revisionen, für Fragen des Berufsprofils oder zur Mitarbeit in Kommissionen zur Verfügung.

Alljährlich werden für die ChemieDiplomanden aller Ingenieurschulen die SVCT-Preise zur Verfügung gestellt, sei es zur Auszeichnung hervorragender Diplomarbeiten oder zur Verwendung in anderer, den Diplomanden zugute kommender Form.

Von wesentlicher Bedeutung sind auch die Beziehungen mit anderen Berufsverbänden. In der Zusammenarbeit mit solchen nimmt insbesondere jene mit dem Schweizerischen Chemiker-Verband (SChV) eine besondere Stelle ein, gehören doch zahlreiche SVCT-Mitglieder gleichzeitig auch dem SChV an. Zudem sind in dessen Vorstand traditionellerweise auch Vorstandsmitglieder des SVCT vertreten. Diese enge Verflechtung gestattet es beiden Verbänden, geschlossen und gemeinsam Interessen der schweizerischen Chemikerschaft koordiniert und erfolgreich zu vertreten. Aus diesem Grund unterstützt die SVCT auch die gegenwärtigen Bestrebungen des $\mathrm{SChV}$ für einen weiteren Zusammenschluss möglichst vieler Chemikervereinigungen der Schweiz und den Ausbau der ausländischen Kontakte.

Über den Chemiesektor hinaus pflegt die SVCT aber seit jeher und zunehmend Beziehungen und gemeinschaftliche Unternehmungen mit anderen HTL-Berufsorganisationen wie dem Schweizerischen Technischen Verband, dem DAVES (Dachverband von HTL-Organisationen) oder zu Ehemaligenvereinen der Ingenieurschulen. Der Ausbau von Kontakten zu solchen, aber auch zu anderen Institutionen im technisch-beruflichen Umfeld wie

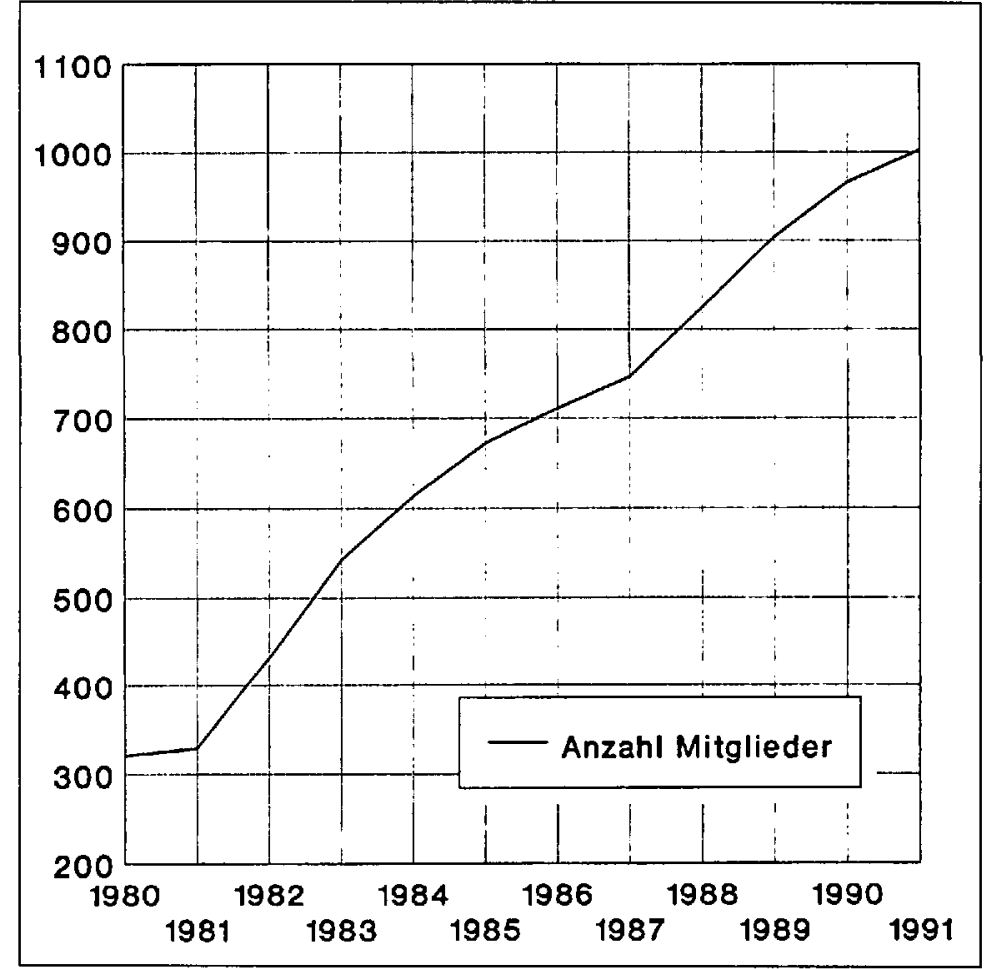

Fig. 3. Schweizerische Vereinigung dipl. Chemiker HTL (SVCT) Entwicklung des Mirgliederbestandes

z.B. zur Akademie der Technischen Wissenschaften (SATW), der Schweizerischen Gesellschaft für Chemische Industrie (SGCI), etc., dürfte in Zukunft vermehrt Thema der SVCT-Verbandsarbeit sein, sind doch letzlich Fragen der Chemie und der Technik deren wie unser gemeinsamer Nenner.

\section{Wie ist die SVCT organisiert?}

Die SVCT besitzt klare Regelungen bez. Organisation, Verantwortlichkeiten und Kompetenzen der Verbandsführung. Der Vorstand arbeitet nach dem Delegati- onsprinzip mit selbständig entscheidenden Funktionsbereichen, sowie mit definierten Stellvertretungen und mittels formulierten Zielen und Aufgabenstellungen. Zur Bewältigung dieser Aufgaben umfasst der Vorstand fünf Ressorts sowie eine Geschäftsleitung (Fig. 5) und als davon unabhängige Kontrollstelle das Rechnungsrevisorat. Daneben besitzt die SVCT ein Sekretariat in der französischsprachigen Schweiz und eine Kontaktstelle im Tessin.

Mitglieder können als Berater oder in Projektgruppen aktiv an den Aufgaben und Tätigkeiten des Verbandes mitzuarbeiten.

\section{Messung und Verarbeitung physikalisch-chem. Messgrössen in der chemischen Industrie \\ 1971 Prozess-Gaschromatographie \\ 1974 Moderne Sicherheitsuntersuchungen in der chem. Industrie/Bedeutung für die Betriebspraxis \\ 1978 Erfassung von Schadstoffexpositionen von Mitarbeitern während der Arbeit \\ 1981 Qualitätssicherung und Produktehaftpflicht \\ 1984 Chemie - Mensch - Computer \\ 1987 Sensortechnologie - neue Perspektiven für die Chemie? \\ 1988 Die Luftreinhalteverordnung der Schweiz (LRV): Konsequenzen und Massnahmen für die schweizerische Industrie \\ 1990 Neue Werkstoffe - Bausteine für die Zukunft \\ 1990 Abfallszene Schweiz: Vorsorge, Recycling, Entsorgung \\ 1991 Sicherheit in der chemischen Industrie}

Schweizerische Vereinigung dipl. Chemiker HTL (SVCT) Postfach 46, 4007 Basel

\section{Geschäftsleitung}

Präsident:

U. Girard, Ciba-Geigy AG, Base

Vizepräsident:

N. di Menna, Umweltschutzamt TG, Frauenfeld

Ressorts

Finanzen:

R.-F. Allegri, Med. Labor Dr. Gubler, Basel

Berufspolitik:

M. Pfammatter, Stabsstelle Umweltschutz, Allschwil

$B$. Zigerlig, $A B B$ infocom, Baden

Mitgliedersekretariat:

H.R. Ott, Sandoz AG, Basel

Information, Kontakte:

R. Pedrazzi, Sandoz AG, Basel

Anlässe, Veranstaltungen:

J.C. Brocher, Sandoz AG, Basel 